\title{
Tendência temporal da sífilis em gestante e congênita em municípios de médio porte
}

\section{do estado do Paraná: 2007-2017}

Temporal trend of the gestacional and congenital syphilis in medium-sized cities in the state of

Paraná: 2007-2017

Tendencia temporal de la sífilis en gestantes y congéneres en municipios medianos del estado de

Paraná: 2007-2017

Recebido: 31/01/2021 | Revisado: 05/02/2021 | Aceito: 08/02/2021 | Publicado: 18/02/2021

Caroliny Stocco

ORCID: https://orcid.org/0000-0002-8327-3084 Universidade Estadual de Ponta Grossa, Brasil E-mail: carolinystocco@hotmail.com Erildo Vicente Müller

ORCID: https://orcid.org/0000-0003-4643-056X Universidade Estadual de Ponta Grossa, Brasil E-mail: erildomuller@hotmail.com

Pollyanna Kássia de Oliveira Borges ORCID: https://orcid.org/0000-0002-9390-0459 Universidade Estadual de Ponta Grossa, Brasil E-mail: pollyannakassia@hotmail.com

\begin{abstract}
Resumo
O objetivo do presente estudo foi descrever a tendência temporal dos casos notificados de sífilis em gestante e congênita em residentes nos municípios de médio porte do estado do Paraná no período de 2007 a 2017. Trata-se de um estudo epidemiológico descritivo e analítico do tipo séries temporais com dados secundários obtidos no DATASUS do SINAN Net e do SINASC. Para verificar a tendência da taxa de detecção de sífilis em gestante e da taxa de incidência de sífilis congênita foi ajustado um modelo de efeitos mistos com efeito aleatório no intercepto e também na inclinação. Também foi utilizada a Regressão Binomial Negativa e o método da Quase-Verossimilhança. Verificou-se influência significativa do tempo sobre a taxa de detecção de sífilis em gestante e na taxa de incidência de sífilis congênita, sendo que a cada ano ocorreu aumento de 31\% (IC 95\% 23-39; p<0,001) e 25\% (IC 95\% 17-34; $\mathrm{p}<0,001)$ nas taxas, respectivamente. A maioria dos municípios apresentou tendência de aumento nas taxas de sífilis em gestante e congênita. Diante disso, se faz necessário adotar medidas de intervenção para mudar a realidade apresentada.
\end{abstract}

Palavras-chave: Sífilis; Gestantes; Sífilis congênita; Infecções sexualmente transmissíveis; Qualidade da assistência à saúde.

\begin{abstract}
The aim of the present study was to describe the temporal trend of the reported cases of gestational and congenital syphilis in residents of medium-sized cities in the State of Paraná between 2007 and 2017. This is a descriptive and analytical epidemiological study of time series type with secondary data obtained in the DATASUS of the SINAN Net and the SINASC. To verify the rate trend of syphilis detection in pregnant women and the incidence rate of congenital syphilis, a mixed effects model with random effect in the intercept and also in the inclination was adjusted. Negative Binomial Regression and the Maximum Likelihood method were also used. There was a significant influence of time on the rate of syphilis detection in pregnant women and on the incidence rate of congenital syphilis, with an increase each year of 31\% (IC 95\% 23-39, p <0.001) and 25\% (IC 95\% 17-34, p <0.001) in the rates, respectively. Most municipalities presented a growing trend in syphilis rates in pregnant and congenital. Given this, it is necessary to adopt intervention measures to change the reality presented.
\end{abstract}

Keywords: Syphilis; Pregnant women; Congenital syphilis; Sexually transmitted infections; Quality of Health Care.

\section{Resumen}

El objetivo de este estudio fue describir la tendencia temporal de los casos notificados de sífilis en embarazadas y congéneres en residentes de municipios de tamaño medio del estado de Paraná desde 2007 hasta 2017. Se trata de un estudio epidemiológico descriptivo y analítico de tipo serie temporal con datos secundarios obtenidos de DATASUS de SINAN Net y SINASC. Para verificar la tendencia de la tasa de detección de la sífilis en las embarazadas y la tasa 
de incidencia de la sífilis congénita, se ajustó un modelo de efectos mixtos con efecto aleatorio sobre la interceptación y también sobre la inclinación. También se utilizó la Regresión Binomial Negativa y el método de Probabilidad Casi Inmediata. Hubo una influencia significativa del tiempo en la tasa de detección de la sífilis en las embarazadas y en la tasa de incidencia de la sífilis congénita, y cada año hubo un aumento del 31\% (IC 95\% 23-39; p<0,001) y del 25\% (IC 95\% 17-34; p<0,001) en las tasas, respectivamente. La mayoría de los municipios mostraron una tendencia al aumento de las tasas de sífilis en mujeres embarazadas y congénitas. Por lo tanto, es necesario adoptar medidas de intervención para cambiar la realidad presentada.

Palabras clave: Sífilis; Mujeres embarazadas; Sífilis congénita; Infecciones de transmisión sexual; Calidad de la atención sanitaria.

\section{Introdução}

Segundo a Organização Mundial de Saúde, mundialmente é estimada a ocorrência de mais de um milhão de casos de Infecções Sexualmente Transmissíveis (IST) por dia, entre elas a sífilis (Brasil, 2018). Também se estima a ocorrência de um milhão de casos de sífilis em gestantes por ano, no mundo (Organización Mundial de la Salud, 2015).

A sífilis é uma doença infecciosa sistêmica, de evolução crônica, causada pela espiroqueta Treponema pallidum (Amsalu, Ferede e Assegu, 2018; Brasil, 2017). A doença tem como principal via de transmissão o contato sexual, sem o uso do preservativo (Secretaria de Estado da Saúde, 2016). Também pode ser transmitida via placentária, a qual pode ocorrer em qualquer momento da gestação ou estágio clínico da doença em gestante não tratada ou inadequadamente tratada (Brasil, 2010).

A ocorrência da sífilis em gestantes pode causar morbidade e mortalidade perinatal significativas que poderiam ser evitadas com a realização dos testes sorológicos no pré-natal, de forma a garantir o tratamento oportuno da gestante e dos parceiros sexuais (Newman et al., 2013). Sendo assim, a incidência de sífilis congênita é um indicativo de falhas na assistência do pré-natal (Cooper et al., 2016; Nonato \& Guimarães, 2015).

No Brasil, entre 2010 e 2017, a taxa de detecção de sífilis em gestante passou de 3,5 para 17,2/1.000 nascidos vivos. E a taxa de incidência de sífilis congênita sofreu aumento de 2,4 para 8,6/1.000 nascidos vivos (Brasil, 2018). Já no Paraná, entre 2010 e 2017, a taxa de detecção de sífilis em gestante passou de 2,2/1.000 nascidos vivos para 16,4/1.000 nascidos vivos e a taxa de incidência de sífilis congênita passou de 1,0 para 5,7/1.000 nascidos vivos (Brasil,2018). Essas informações mostram que a sífilis em gestante e congênita permanecem como um grande problema de saúde pública.

Assim, o objetivo do presente estudo foi descrever a tendência temporal dos casos notificados de sífilis em gestante e congênita em residentes nos municípios de médio porte do Estado do Paraná no período de 2007 a 2017.

\section{Metodologia}

Este estudo tem uma natureza quantitivo e consiste de um estudo epidemiológico descritivo e analítico do tipo séries temporais (Pereira et al., 2018), com dados secundários obtidos no TabNet do DATASUS do Sistema de Informação de Agravos de Notificação (SINAN Net) de sífilis em gestante (Datasus, 2021a) e congênita (Datasus, 2021b). Foram selecionados os casos de residentes nos municípios de médio porte do estado do Paraná, notificados entre os anos de 2007 a 2017.

Segundo Stamm et al. (2013) as cidades brasileiras consideradas como de médio porte são aquelas cuja população urbana situa-se entre 100 mil a 500 mil habitantes. Assim, foram elencados dezesseis municípios, com base na população do Censo 2010 (Datasus, 2021c): Almirante Tamandaré (103.204 habitantes), Apucarana (120.919 habitantes), Arapongas (104.150 habitantes), Araucária (119.123 habitantes), Campo Largo (112.377 habitantes), Cascavel (286.205 habitantes), Colombo (212.967 habitantes), Foz do Iguaçu (256.088 habitantes), Guarapuava (167.328 habitantes), Maringá (357.077 habitantes), Paranaguá (140.469 habitantes), Pinhais (117.008 habitantes), Ponta Grossa (311.611 habitantes), São José dos 
Pinhais (264.210 habitantes), Toledo (119.313 habitantes) e Umuarama (100.676 habitantes). Optou-se em pesquisar os municípios de porte médio, porque os mesmos são referência e polos de oferta de serviços de saúde para os municípios menores em suas respectivas áreas de abrangência.

Para o cálculo da taxa de detecção de sífilis em gestante foi utilizado o número de casos notificados no SINAN Net, por ano no local de residência, dividido pelo número de nascidos vivos do mesmo ano no local de residência e multiplicado por 1.000. Para o cálculo da taxa de incidência da sífilis congênita foi utilizado o número de casos novos em menores de um ano obtidos do SINAN Net, por ano no local de residência, dividido pelo número de nascidos vivos do mesmo ano no local de residência e multiplicado por 1.000. O número de nascidos vivos de cada município por ano foi obtido no TABNET do Departamento de Informática do Sistema Único de Saúde (DATASUS) por meio do Sistema de Informações sobre Nascidos Vivos (SINASC) para os anos de 2007 a 2016 (Datasus, 2021d). Tendo em vista a indisponibilidade do dado para o ano de 2017 na base do DATASUS, o mesmo foi obtido a partir do TABNET da Secretaria de Estado da Saúde do Paraná (Paraná, 2021).

Para avaliar as taxas de detecção de sífilis em gestante e a taxa de incidência de sífilis congênita dos munícipios de médio porte do estado do Paraná, foram utilizados modelos de efeitos mistos (Fitzmaurice, Laird \& Ware, 2012). E para verificar a tendência da taxa de detecção de sífilis em gestante e da taxa de incidência de sífilis congênita foi ajustado um modelo de efeitos mistos com efeito aleatório no intercepto e também na inclinação (Pinheiro \& Bates, 2000). Dessa forma, o efeito aleatório do intercepto representa a taxa de sífilis, enquanto que o efeito aleatório na inclinação indica o efeito da tendência da taxa ao longo do tempo. O sujeito foi cada município em cada momento no tempo, o que permite verificar o efeito do tempo sobre as taxas de sífilis em cada município.

Para modelagem das taxas optou-se por utilizar a Regressão Binomial Negativa e o método da QuaseVerossimilhança (Mccullagh \& Nelder, 1989) para a estimação do modelo, possibilitando assim, a estimação de variâncias robustas.

Cabe ressaltar que o tempo foi centrado em 2017 para evitar a estrutura de correlação entre os efeitos aleatórios. Com o tempo centrado em 2017, o coeficiente (Intercepto) significa o valor médio esperado para a variável resposta de interesse no ano de 2017 para todos os municípios, enquanto que fornece o quanto o j-ésimo município está acima ou abaixo da média geral da variável resposta de interesse. O coeficiente fornece, em média, a tendência da variável resposta de interesse a cada um ano acrescido, enquanto que fornece, em média, a tendência para a variável resposta de interesse ao longo do tempo para o j-ésimo município. Este modelo é conhecido como modelo de efeitos mistos, pois se tem o efeito fixo ( ) e o efeito aleatório ( . A notação j significa $\mathrm{j}=1,2, \ldots, 16$ (municípios) e o tempo $(\mathrm{t})$ variando de 2007 a 2017 . A notação significa o tempo $\mathrm{t}$ avaliado para o j-ésimo município.

O software utilizado nas análises estatísticas e para a espacialização foi o R (versão 3.5.1).

O projeto da pesquisa foi submetido ao Comitê de Ética da Universidade Estadual de Ponta Grossa (UEPG) e aprovado sob o parecer $\mathrm{n}^{\mathrm{o}} 2.305 .861$ em 29/09/2017.

\section{Resultados}

Com relação a sífilis em gestante, entre 2007 e 2017 foram notificados 3.529 casos em municípios de médio porte do Estado do Paraná. No período, a taxa de detecção média de sífilis em gestante foi de 7,6 para cada 1.000 nascidos vivos.

Em 2007, o município de Apucarana, Arapongas, Paranaguá, São José dos Pinhais e Umuarama não apresentaram casos de sífilis em gestante. O município de Toledo foi o que teve a maior taxa de detecção, com 3,5 casos para cada 1.000 
nascidos vivos, seguido pelos municípios de Almirante Tamandaré, Cascavel e Pinhais com taxa de 1,7 casos para cada 1.000 nascidos vivos.

Em 2017, o município de Toledo apresentou a maior taxa de detecção com 23,1 casos para cada 1.000 nascidos vivos, representando um aumento de 6,6 vezes em relação a 2007, seguido pelo município de Cascavel com 18,6 casos para cada 1.000 nascidos vivos, representando um aumento de 11,1 vezes em relação a 2007.

A análise de tendência da taxa de detecção de sífilis em gestante mostrou influência significativa $(\mathrm{p}<0,001)$ do ano sobre essa taxa, sendo que em um mesmo município, a cada um ano que se passa, ocorre um aumento de 31\% (IC95\% 23-39) na taxa de sífilis em gestante. A maioria dos municípios obteve uma taxa de detecção igual à taxa média geral. Todos os municípios apresentaram tendência de crescimento da taxa, com exceção de Paranaguá que apresentou tendência constante (Tabela 1).

Tabela 1 - Análise de tendência da taxa de detecção de sífilis em gestante em municípios de médio porte do estado do Paraná.

\begin{tabular}{|c|c|c|c|c|c|c|}
\hline \multirow[b]{2}{*}{ Município } & \multicolumn{3}{|c|}{ Intercepto } & \multicolumn{3}{|c|}{ Inclinação } \\
\hline & $\operatorname{Exp}\left(\alpha_{j}\right)$ & IC $-95 \%$ & Tendência & $\begin{array}{c}\operatorname{Exp} \\
\left(\beta_{1}+\mu_{j}\right)\end{array}$ & IC $-95 \%$ & Tendência \\
\hline Almirante Tamandaré & 1,41 & {$[0,91 ; 2,21]$} & Igual a média & 1,24 & {$[1,11 ; 1,39]$} & Crescente \\
\hline Apucarana & 1,43 & {$[0,88 ; 2,34]$} & Igual a média & 1,35 & {$[1,16 ; 1,58]$} & Crescente \\
\hline Arapongas & 0,79 & {$[0,48 ; 1,32]$} & Igual a média & 1,31 & {$[1,12 ; 1,53]$} & Crescente \\
\hline Araucária & 1,16 & {$[0,74 ; 1,82]$} & Igual a média & 1,30 & {$[1,16 ; 1,46]$} & Crescente \\
\hline Campo Largo & 0,43 & {$[0,25 ; 0,73]$} & Abaixo da média & 1,25 & {$[1,09 ; 1,43]$} & Crescente \\
\hline Cascavel & 1,52 & {$[1,00 ; 2,33]$} & Igual a média & 1,36 & {$[1,22 ; 1,50]$} & Crescente \\
\hline Colombo & 1,40 & {$[0,91 ; 2,14]$} & Igual a média & 1,28 & {$[1,16 ; 1,42]$} & Crescente \\
\hline Foz do Iguaçu & 1,11 & {$[0,71 ; 1,72]$} & Igual a média & 1,49 & {$[1,33 ; 1,67]$} & Crescente \\
\hline Guarapuava & 0,42 & {$[0,26 ; 0,67]$} & Abaixo da média & 1,16 & {$[1,02 ; 1,32]$} & Crescente \\
\hline Maringá & 1,29 & {$[0,83 ; 1,99]$} & Igual a média & 1,47 & {$[1,31 ; 1,64]$} & Crescente \\
\hline Paranaguá & 0,62 & {$[0,39 ; 0,99]$} & Abaixo da média & 1,03 & {$[0,91 ; 1,15]$} & Constante \\
\hline Pinhais & 1,20 & {$[0,77 ; 1,87]$} & Igual a média & 1,29 & {$[1,16 ; 1,44]$} & Crescente \\
\hline Ponta Grossa & 1,17 & {$[0,76 ; 1,79]$} & Igual a média & 1,39 & {$[1,25 ; 1,55]$} & Crescente \\
\hline São José dos Pinhais & 1,32 & {$[0,84 ; 2,07]$} & Igual a média & 1,56 & {$[1,38 ; 1,76]$} & Crescente \\
\hline Toledo & 1,18 & {$[0,75 ; 1,85]$} & Igual a média & 1,26 & {$[1,13 ; 1,40]$} & Crescente \\
\hline Umuarama & 0,73 & {$[0,44 ; 1,22]$} & Igual a média & 1,28 & {$[1,10 ; 1,50]$} & Crescente \\
\hline
\end{tabular}

Fonte: Autores (2019).

A Figura 1 mostra a representação do crescimento da taxa de detecção de sífilis em gestante ao longo do tempo por município. Todos os municípios, exceto o município de Paranaguá, apresentaram tendência de aumento da taxa de sífilis em gestante. 
Figura 1 - Representação do efeito da tendência da taxa de detecção de sífilis em gestante ao longo do tempo nos municípios de médio porte do Paraná, 2007 a 2017.

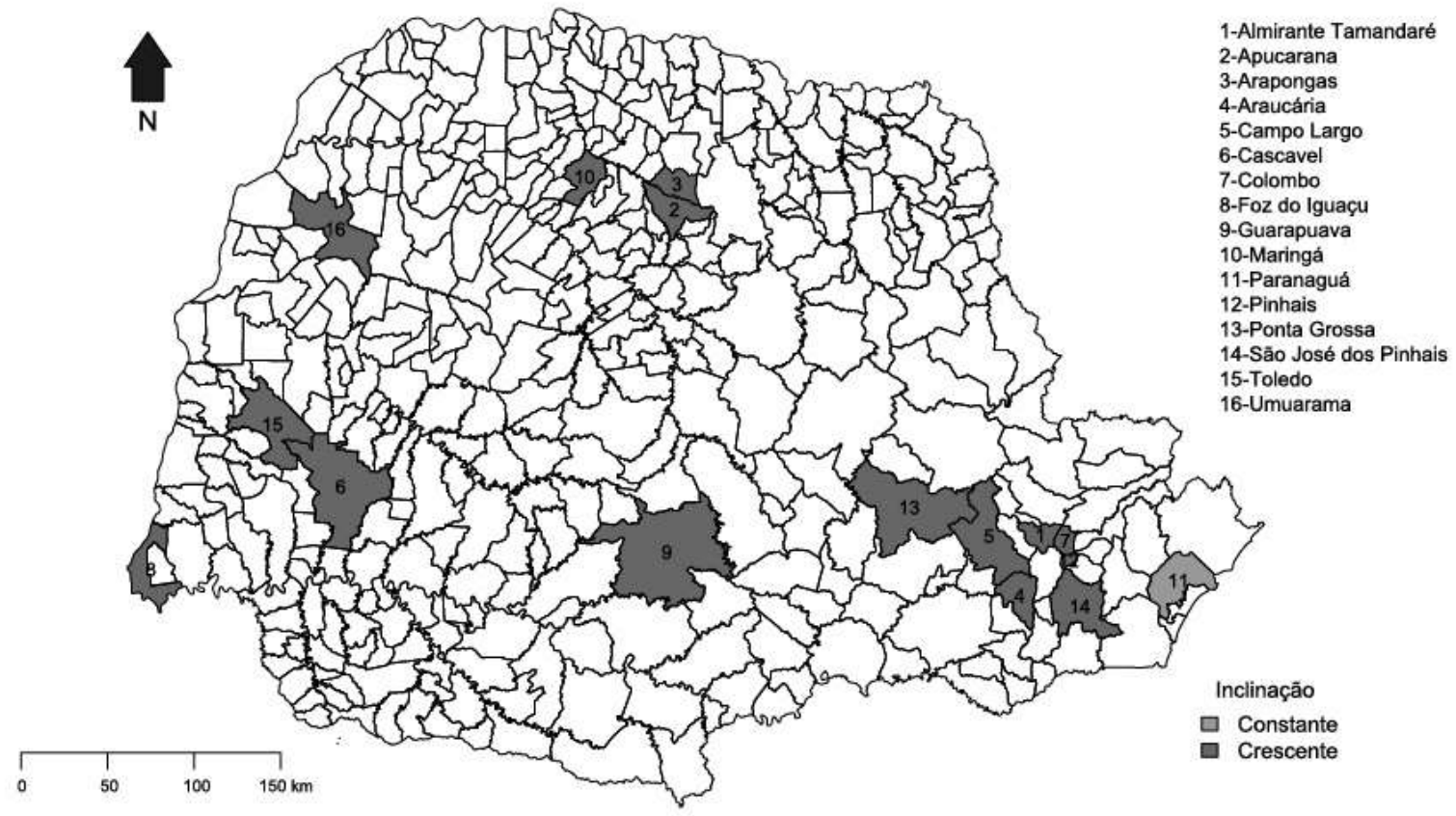

Fonte: Autores (2019).

Quanto à sífilis congênita, entre 2007 e 2017, foram notificados 1.656 casos em residentes nos municípios de médio porte do Estado do Paraná. No período, a taxa de incidência média de sífilis congênita foi de 4,3 para cada 1.000 nascidos vivos.

Em 2007, os municípios Apucarana, Arapongas, Araucária, Campo Largo, Maringá, Paranaguá, Pinhais, Ponta Grossa, São José dos Pinhais e Toledo não apresentaram casos de sífilis congênita. O município de Almirante Tamandaré foi o que apresentou a maior incidência, com 1,7 casos para cada 1.000 nascidos vivos, seguido pelo município de Umuarama com 0,8 casos para cada 1.000 nascidos vivos.

Em 2017, o município de Pinhais apresentou a maior incidência de sífilis congênita com 17,5 casos para cada 1.000 nascidos vivos, seguido pelo município de Almirante Tamandaré com 16,8 casos para cada 1.000 nascidos vivos, representando um aumentou de 9,9 vezes da taxa para o último município.

Vale ressaltar que o município de São José dos Pinhais foi o que apresentou a menor incidência de sífilis congênita de todo o período, com 0,2 casos para cada 1.000 nascidos vivos em 2008 e 2010. No entanto, para o ano de 2016 verificou-se aumento considerável para 19,7 casos para cada 1.000 nascidos vivos, sendo também a maior taxa de incidência observada dentre os municípios.

E ainda, que o município de Araucária e Guarapuava apresentaram subnotificação de casos de sífilis em gestante em 2009, já que houve notificação de casos de sífilis congênita para o mesmo ano. Assim como o município de Umuarama em 2007,2008 e 2011.

A análise de tendência da taxa de incidência de sífilis congênita mostrou que houve influência significativa $(\mathrm{p}<0,001)$ do ano sobre a taxa de sífilis congênita, sendo que em um mesmo município, a cada um ano que se passa, ocorreu aumento de $25 \%$ (IC 95\% 17-34) na taxa de sífilis.

Os municípios Almirante Tamandaré, Apucarana, Colombo, Maringá, Paranaguá, Pinhais e São José dos Pinhais apresentaram uma taxa de incidência de sífilis congênita maior que a taxa média geral de sífilis congênita. Os municípios 
Cascavel, Guarapuava, Ponta Grossa e Toledo tiveram uma taxa menor que a taxa média geral. A maioria dos municípios apresentou tendência de crescimento da taxa de sífilis congênita, com exceção do município de Apucarana e Toledo que apresentaram tendência constante (Tabela 2).

Tabela 2 - Análise de tendência da taxa de incidência sífilis congênita em municípios de médio porte do estado do Paraná.

\begin{tabular}{cccc|ccc}
\hline & \multicolumn{3}{c|}{ Intercepto } & \multicolumn{3}{c}{ Inclinação } \\
\cline { 2 - 7 } Município & $\mathbf{E x p}\left(\boldsymbol{\alpha}_{j}\right)$ & $\mathbf{I C} \mathbf{- 9 5 \%}$ & Tendência & $\begin{array}{c}\text { Exp } \\
\left(\boldsymbol{\beta}_{1+\boldsymbol{\mu}}\right)\end{array}$ & $\mathbf{I C ~ - ~ 9 5 \%}$ & Tendência \\
\hline Almirante Tamandaré & 2,38 & {$[1,55 ; 3,65]$} & Acima da média & 1,19 & {$[1,06 ; 1,33]$} & Crescente \\
Apucarana & 2,11 & {$[1,31 ; 3,40]$} & Acima da média & 1,16 & {$[0,97 ; 1,38]$} & Constante \\
Arapongas & 0,72 & {$[0,40 ; 1,29]$} & Igual a média & 1,28 & {$[1,06 ; 1,54]$} & Crescente \\
Araucária & 0,84 & {$[0,51 ; 1,39]$} & Igual a média & 1,17 & {$[1,02 ; 1,34]$} & Crescente \\
Campo Largo & 0,57 & {$[0,31 ; 1,06]$} & Igual a média & 1,21 & {$[1,03 ; 1,43]$} & Crescente \\
Cascavel & 0,62 & {$[0,39 ; 0,97]$} & Abaixo da média & 1,23 & {$[1,09 ; 1,37]$} & Crescente \\
Colombo & 1,81 & {$[1,20 ; 2,71]$} & Acima da média & 1,22 & {$[1,10 ; 1,36]$} & Crescente \\
Foz do Iguaçu & 1,05 & {$[0,67 ; 1,65]$} & Igual a média & 1,37 & {$[1,19 ; 1,58]$} & Crescente \\
Guarapuava & 0,32 & {$[0,18 ; 0,58]$} & Abaixo da média & 1,18 & {$[1,02 ; 1,38]$} & Crescente \\
Maringá & 2,49 & {$[1,62 ; 3,83]$} & Acima da média & 1,57 & {$[1,36 ; 1,81]$} & Crescente \\
Paranaguá & 1,60 & {$[1,01 ; 2,52]$} & Acima da média & 1,23 & {$[1,09 ; 1,40]$} & Crescente \\
Pinhais & 1,64 & {$[1,04 ; 2,59]$} & Acima da média & 1,28 & {$[1,13 ; 1,45]$} & Crescente \\
Ponta Grossa & 0,25 & {$[0,15 ; 0,43]$} & Abaixo da média & 1,17 & {$[1,01 ; 1,35]$} & Crescente \\
São José dos Pinhais & 3,63 & {$[2,41 ; 5,45]$} & Acima da média & 1,58 & {$[1,40 ; 1,78]$} & Crescente \\
Toledo & 0,41 & {$[0,23 ; 0,73]$} & Abaixo da média & 1,08 & {$[0,94 ; 1,25]$} & Constante \\
Umuarama & 0,63 & {$[0,31 ; 1,25]$} & Igual a média & 1,18 & {$[1,02 ; 1,38]$} & Crescente \\
\hline
\end{tabular}

Fonte: Autores (2019).

A Figura 2 mostra a representação do crescimento da taxa de incidência de sífilis congênita ao longo do tempo por município. Todos os municípios, exceto os municípios de Apucarana e Toledo, apresentaram tendência de crescimento da taxa de sífilis congênita. 
Figura 2 - Representação da tendência da taxa de incidência de sífilis congênita ao longo do tempo nos municípios de médio porte do Paraná, 2007 a 2017.

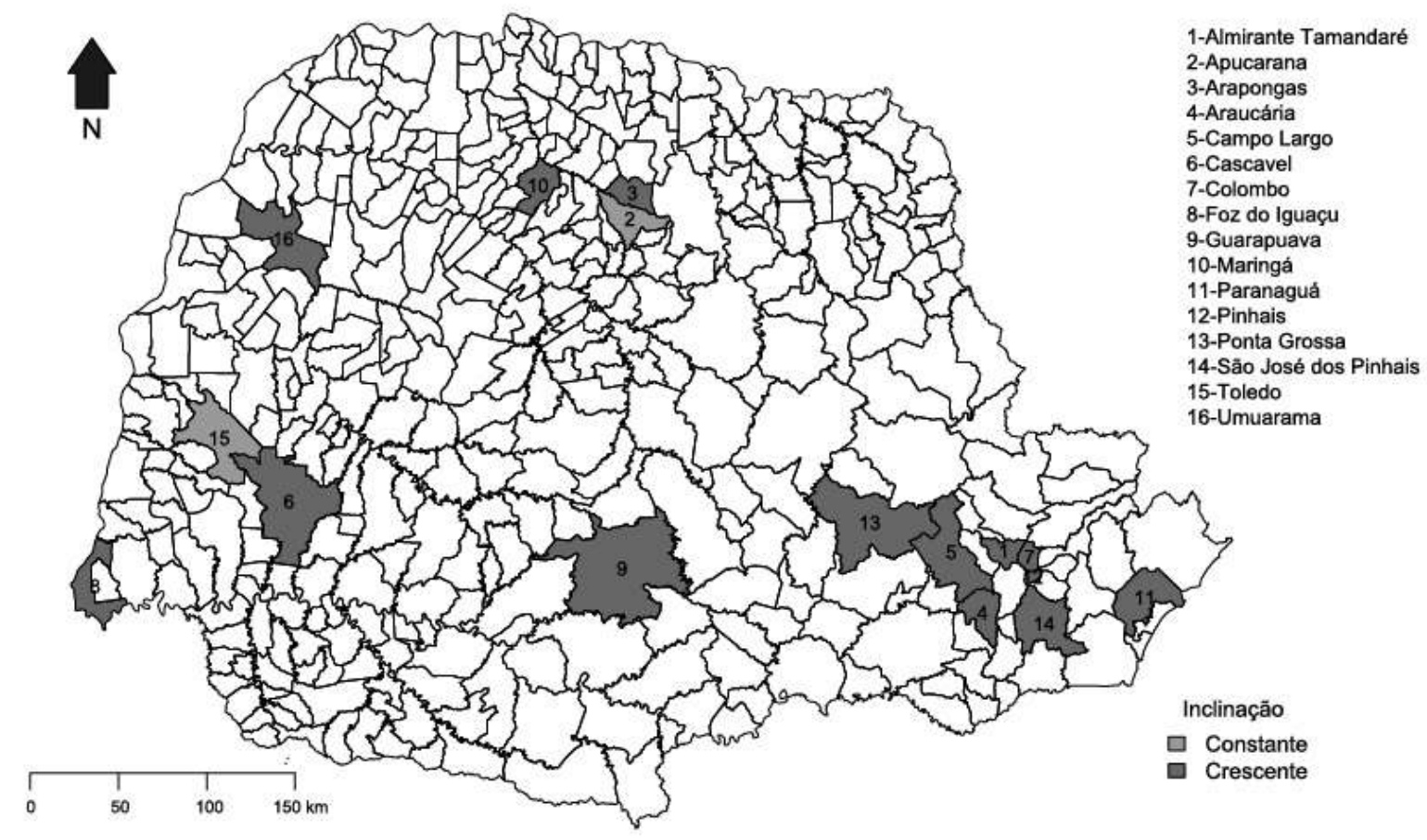

Fonte: O autor, 2019.

\section{Discussão}

Os principais achados deste estudo mostram que, entre 2007 e 2017, houve aumento tanto das taxas de detecção de sífilis em gestante quanto das taxas de incidência de sífilis congênita na maioria dos municípios de médio porte do estado do Paraná. Todos os municípios apresentaram tendência de crescimento da taxa de detecção de sífilis em gestante, com exceção de Paranaguá que apresentou tendência constante. A maioria dos municípios apresentou tendência de aumento da taxa de sífilis congênita, com exceção dos municípios de Apucarana e Toledo os quais apresentaram tendência constante.

No Brasil, entre 2010 e 2017, a taxa de detecção de sífilis em gestante apresentou aumento de 4,9 vezes, passando de 3,5 para 17,2/1.000 nascidos vivos. E a taxa de incidência de sífilis congênita demonstrou aumento de 3,6 vezes, passando de 2,4 para 8,6/1.000 nascidos vivos (Brasil, 2018).

Nos Estados Unidos, entre 2008 e 2012 houve redução da taxa de sífilis congênita de 10,5 para 8,4/100.000 nascidos vivos. Porém, a partir de 2013 a taxa sofreu aumentou a cada ano. Em 2014 passou para 11,6/100.000 nascidos vivos. Em 2017 a taxa chegou a 23,7/100.000 nascidos vivos e em 2018 alcançou 33,1/100.000 nascidos vivos, representando um aumento de $39,7 \%$ em relação a 2017. O aumento dessa taxa coincide com o aumento das taxas de sífilis primária e secundária entre mulheres em idade fértil entre 2014 e 2018 (CDC, 2019). Apesar do aumento encontrado no país, as taxas são muito mais baixas que a do Brasil, já que a constante utilizada para o cálculo foi por 100.000 nascidos vivos.

Na América Latina, entre 2011 e 2012, as menores taxas de incidência de sífilis congênita encontradas dentre os países foi em Honduras e Argentina, com 1,0 e 8,1/1.000 nascidos vivos, respectivamente. Já a Bolívia e Panamá foram os países que apresentaram as maiores taxas, com 75,6 e 108,0/1.000 nascidos vivos (Serruya et al., 2015).

Já no estado do Paraná, entre 2010 e 2017, a taxa de detecção de sífilis em gestante passou de 2,2/1.000 nascidos vivos para 16,4/1.000 nascidos vivos (Brasil, 2018), representando um aumento de 7,4 vezes. E a taxa de incidência de sífilis congênita passou de 1,0 para 5,7/1.000 nascidos vivos, representando um aumento de 5,7 vezes (Brasil, 2018). No presente 
estudo Toledo foi o município que apresentou a maior taxa de detecção de sífilis em gestante, representando um aumento de 6,6 vezes entre 2007 a 2017. Quanto à incidência de sífilis congênita, Almirante Tamandaré aumentou a taxa em 9,9 vezes no mesmo período. Portanto, o padrão de crescimento na detecção da sífilis em gestante, bem como a incidência da sífilis congênita dos municípios de médio porte do Paraná, vem acompanhando os indicadores nacionais e estaduais. Porém, alguns municípios ultrapassaram estes indicadores e apresentaram taxas bem mais elevadas, quando comparadas ao estado ou o Brasil.

Em 2012, a Secretaria de Estado da Saúde do Paraná implantou a Rede Mãe Paranaense (RMP) com o objetivo de organizar a atenção materno-infantil (Paraná, 2017) através da captação precoce da gestante, da realização de no mínimo 7 consultas de pré-natal, a realização de exames, a estratificação de risco das gestantes e das crianças, o atendimento em ambulatório especializado para as gestantes e crianças de risco e a garantia do parto por meio de um sistema de vinculação ao hospital conforme o risco gestacional (Paraná, 2018). Assim, esperava-se a constância ou queda na sífilis gestacional e congênita nos municípios de médio porte do Paraná, após a implantação da política pública da RMP.

No entanto, estudo realizado no estado do Rio Grande do Sul que avaliou a tendência temporal da sífilis congênita entre 2001 e 2012 revelou que o aumento das taxas de incidência não está relacionado ao aumento do número de casos, mas sim devido à melhoria do diagnóstico e notificação dos casos, já que houve correlação positiva e significativa entre o aumento das equipes de Estratégia de Saúde da Família e as taxas de incidência de sífilis congênita e de detecção da sífilis em gestantes (Teixeira et al., 2018).

No estado de Minas Gerais, outra pesquisa que avaliou a evolução temporal da sífilis congênita e sífilis em gestantes entre 2007 e 2015, apresentou tendência crescente $(\mathrm{p}<0,05)$ de 0,99 casos ao ano de sífilis em gestantes e de 0,98 casos da incidência de sífilis congênita, com aumento considerável a partir de 2011. Autores descrevem que o aumento na incidência pode estar relacionado à redução de subnotificações ou ainda com a melhoria da atenção a mulher na gestação após a implantação da Rede Cegonha em 2011 (Alves, 2017).

Em Santa Catarina a análise da tendência temporal da incidência da sífilis congênita entre 2007 a 2016 mostrou que houve aumento de $1190 \%$ das taxas de incidência de sífilis congênita no estado, com incremento médio anual de 0.61 casos na taxa (Rocha \& Magajewski, 2018).

No estado do Sergipe foi analisada a tendência temporal da sífilis congênita entre 2007 a 2015, tendo encontrado tendência de crescimento de incidência de sífilis congênita entre 2007 a 2010 e uma estabilização da taxa entre 2011 e 2015 (Silveira, 2018).

Em Porto Velho - TO no ano de 2009 a incidência de sífilis congênita era de 0,92/1.000 nascidos vivos e passou para 8,65/1.000 nascidos vivos em 2014. E no estado do Tocantins a incidência da doença era de 0,5/1.000 nascidos vivos em 2009 e passou para 4,21/1.000 nascidos vivos em 2014 (Moreira et al., 2017).

Já em Goiás, um estudo realizado com casos de sífilis congênita entre 2008 a 2013 revelou diminuição da taxa de incidência de sífilis congênita no estado no período investigado. Em contrapartida, o estudo discute que a maioria das mães realizou o pré-natal, porém foram diagnosticadas com sífilis no momento do parto/curetagem ou após, e que o(s) parceiro(s) sexuais não foi/foram tratado(s) concomitantemente à gestante (Amâncio et al., 2016).

Os achados do presente estudo corroboram com a maioria dos autores, nos quais foi encontrada tendência de aumento da taxa de detecção de sífilis em gestante e da taxa de incidência de sífilis congênita, com exceção do estudo de Goiás que apresentou diminuição da taxa de incidência de sífilis congênita.

Assim, a transmissão vertical da sífilis permanece como um grande problema de saúde pública, embora o custo da intervenção preventiva seja muito mais baixo que o custo do tratamento da doença (Domingues et al., 2013). No entanto, tanto o presente estudo como outros estudos evidenciaram um aumento na incidência da sífilis congênita. Mesmo ultrapassado o 
prazo estabelecido pela OPAS, que tinha como meta até 2015 reduzir a incidência de sífilis congênita para 0,5 caso por 1.000 nascidos vivos (Organização Pan-Americana da Saúde, 2005), os municípios de médio porte do Paraná em 2017 estavam, quase em sua totalidade, longe do alcance da meta supracitada.

O Ministério da Saúde (Brasil, 2015) aponta que o aumento de casos de sífilis em gestante está relacionado à melhoria das ações de vigilância e ampliação da utilização de teste rápido no pré-natal, através da implantação da Rede Cegonha. Porém, em 2016 foi publicada uma nota técnica ministerial informando sobre a falta de matéria prima para a produção de penicilina benzatina e cristalina, a partir de junho de 2014, o que causou um desabastecimento desses antibióticos a nível nacional (Brasil, 2016). Sendo assim, a falta do medicamento pode ter contribuído para o aumento significativo da incidência da sífilis congênita, já que o esquema alternativo com ceftriaxona não ultrapassa a barreira placentária.

Beck e Souza (2017), comentam que outros estudos apontam que os principais fatores relacionados ao aumento da incidência de sífilis congênita foram falhas nas medidas profiláticas e na assistência pré-natal, tratamento inadequado da gestante, parceiro não tratado ou tratado inadequadamente e falta de documentação terapêutica pré-natal.

Um estudo realizado na cidade de Nova York (estado de Nova York), entre 2010 e 2016, encontrou que em aproximadamente um terço dos casos de sífilis congênita o principal fator contribuinte foi o início tardio do pré-natal e a falta de acesso aos serviços de saúde (Slutsker, Hennessy \& Schillinger, 2018).

É importante destacar que além das questões relacionadas à qualidade da assistência à saúde, estudos discutem que a sífilis congênita também está relacionada a questões demográficas (Cardoso et al., 2018), socioeconômicas (Arnesen, Serruya \& Durán, 2015; Feliz et al, 2016) e comportamentais (Hebmuller, Fiori \& Lago, 2015).

O presente estudo apresentou limitações por utilizar dados secundários, os quais podem estar subnotificados e, consequentemente, ter repercutido em menores taxas de detecção de sífilis em gestante e taxa de incidência de sífilis congênita. Outro estudo que utilizou dados secundários também aponta tal limitação (Antunes \& Cardoso, 2015).

Sugere-se a associação das taxas de deteç̧ão de sífilis em gestante e taxa de incidência de sífilis congênita com outros fatores, tais como dados socioeconômicos, a fim de verificar se a doença também pode estar relacionada com determinantes sociais. E ainda, com dados epidemiológicos para verificar se o aumento das taxas pode estar relacionado com o tratamento inadequado da gestante, com o não tratamento do parceiro concomitantemente à gestante, com o diagnóstico tardio no prénatal, com a não realização dos testes imunológicos no pré-natal ou ainda com o baixo número de consultas de pré-natal. Mais estudos são necessários para o aprofundamento da temática de melhoria das taxas de sífilis em gestante e congênita, além da necessidade de adotar medidas de intervenção para mudar a realidade apresentada.

\section{Referências}

Amâncio, V. C.; Graciano, A. R.; Cozer, A. M.; Assis, L. P. F. \& Dias, D. C. S. (2016). Epidemiologia da sífilis congênita no estado de Goiás. Revista Brasileira de Educação e Saúde, 4 (2): 58-63.

Alves, P. I. C. et al. (2020). Evolução temporal e caracterização dos casos de sífilis congênita e em gestantes em Minas Gerais, 2007 a 2015 . Ciências \& Saúde Coletiva, 25(8): 2949-2960. https://dx.doi.org/10.1590/1413-81232020258.20982018

Amsalu, A.; Ferede, G \& Assegu, D. (2018). High seroprevalence of syphilis infection among pregnant women in Yiregalem hospital southern Ethiopia. BMC Infectious Diseases, 18(109):1-6. https://doi.org/10.1186/s12879-018-2998-8

Antunes, J. L. F. \& Cardoso, M. R. A. (2015). Uso da análise de séries temporais em estudos epidemiológicos. Epidemiologia e Serviços de Saúde, 24 (3): 565-576. http://dx.doi.org/10.5123/S1679-49742015000300024

Arnesen, L.; Serruya, S. \& Durán, P. (2015). Gestational syphilis and stillbirth in the Americas: a systematic review and meta-analysis. Revista Panamericana de Salud Publica, 37 (6): 422-429.

Beck, E. Q. \& Souza, M. (2017). Fatores de risco para sífilis congênita. Disciplinarum Scientia, 18 (2): 419-432.

Brasil. (2010). Ministério da Saúde. Coordenação de Doenças Sexualmente Transmissíveis e Aids. Súfilis: Estratégias para Diagnóstico no Brasil. Brasília: Ministério da Saúde. 100 p. http://bvsms.saude.gov.br/bvs/publicacoes/sifilis_estrategia_diagnostico_brasil.pdf. 
Brasil. (2015) Ministério da Saúde. Secretaria de Vigilância em Saúde. Departamento de DST, Aids e Hepatites Virais. Protocolo clínico e diretrizes terapêuticas para atenção integral às pessoas com infecções sexualmente transmissíveis. Brasília: 120 p. http://bvsms.saude.gov.br/bvs/publicacoes/protocolo_clinico_diretrizes_terapeutica_atencao_integral_pessoas_infeccoes_sexualmente_transmissiveis.pdf.

Brasil. (2016). Ministério da Saúde. Secretaria de Vigilância em Saúde. Departamento de DST, Aids e Hepatites Virais. Nota Informativa ${ }^{\circ}$ 006/2016/GAB/DDAHV/SVS/MS. Informa a respeito da importância e urgência na aquisição de penicilina cristalina (ou potássica), tendo em vista que tal medicamento é tratamento padrão para diversas doenças com relevante impacto em saúde pública. http://www.aids.gov.br/sites/default/files/legislacao/2016/notas_informativas/nota_informativa_no006_importancia_e_urgencia_na_a_82765.pdf.

Brasil. Ministério da Saúde. (2017). Secretaria de Vigilância em Saúde. Coordenação-Geral de Desenvolvimento da Epidemiologia em Serviços. Guia de Vigilância em Saúde: volume único. 2 ed. Brasília: Ministério da Saúde. p. 270-285. http://portalarquivos.saude.gov.br/images/pdf/2017/outubro/06/VolumeUnico-2017.pdf.

Brasil. Ministério da Saúde (2018). Secretaria de Vigilância em Saúde. Departamento de Vigilância, Prevenção e Controle das Infecções Sexualmente Transmissíveis, do HIV/Aids e das Hepatites Virais (DIAHV). Organizadores: Benzaken AS et al. Boletim Epidemiológico de Sífilis. Brasília, v. 49, n. 45, out. 2018. 48p. http://www.aids.gov.br/pt-br/pub/2018/boletim-epidemiologico-de-sifilis-2018.

Cardoso, A. R. P.; Araújo, M. A. L.; Cavalcante, M. S.; Frota, M. A. \& Melo, S. P. (2018). Análise dos casos de sífilis gestacional e congênita nos anos de 2008 a 2010 em Fortaleza, Ceará, Brasil. Ciência \& Saúde Coletiva, 23 (2): 563-574. http://dx.doi.org/10.1590/1413-81232018232.01772016.

CDC. Centers for Disease Control and Prevention. (2019). Sexually Transmitted Disease Surveillance 2018. Atlanta. p. 29. https://www.cdc.gov/std/stats18/STDSurveillance2018-full-report.pdf.

Cooper, J. M.; Michelow, I. C.; Wozniak, P. S. \& Sánchez, P. J. (2016). Em tempo: a persistência da sífilis congênita no Brasil - Mais avanços são necessários. Revista Paulista de Pediatria, 34 (3): 251-253. http://dx.doi.org/10.1016/j.rppede.2016.06.004.

Datasus. (2021a). Departamento de Informática do Sistema Único de Saúde. Informações de saúde (TABNET). Epidemiológicas e morbidade.

Doenças e Agravos de Notificação - De 2007 em diante (SINAN). Sífilis em Gestante - casos confirmados notificados no Sistema de Informação de Agravos de Notificação - Brasil. http://tabnet.datasus.gov.br/cgi/deftohtm.exe?sinannet/cnv/sifilisgestantebr.def.

Datasus. (2021b). Departamento de Informática do Sistema Único de Saúde. Informações de saúde (TABNET). Epidemiológicas e morbidade.

Doenças e Agravos de Notificação - De 2007 em diante (SINAN). Sífilis Congênita - casos confirmados notificados no Sistema de Informação de Agravos de Notificação - Brasil. http://tabnet.datasus.gov.br/cgi/tabcgi.exe?sinannet/cnv/sifilisbr.def.

Datasus. (2021c). Departamento de Informática do Sistema Único de Saúde. Informações de saúde (TABNET). Demográficas e socioeconômicas. Censos (1980, 1991, 2000 e 2010), Contagem (1996) e projeções intercensitárias (1981 a 2012), segundo faixa etária, sexo e situação de domicílio. População residente - Paraná. http://tabnet.datasus.gov.br/cgi/deftohtm.exe?ibge/cnv/poppr.def.

Datasus. (2021d). Departamento de Informática do Sistema Único de Saúde. Informações de saúde (TABNET). Estatísticas vitais. Nascidos vivos - 1994 a 2016. Nascidos vivos - Paraná. http://tabnet.datasus.gov.br/cgi/deftohtm.exe?sinasc/cnv/nvpr.def.

Domingues, R. M. S. M.; Saraceni, V.; Hartz, Z. M. A. \& Leal, M. C. (2013). Sífilis congênita: evento sentinela da qualidade da assistência pré-natal. Revista de Saúde Pública, 47 (11): 147-157. ttp://www.scielo.br/pdf/rsp/v47n1/19.pdf.

Feliz, M. C.; Medeiros, A. R. P.; Rossoni, A. M.; Tahnus, T.; Pereira, A. M. V. B. \& Rodrigues, C. Aderência ao seguimento no cuidado ao recém-nascido exposto à sífilis e características associadas à interrupção do acompanhamento. Revista Brasileira de Epidemiologia, 19 (4): 727-739. http://dx.doi.org/10.1590/1980-5497201600040004.

Fitzmaurice, G. M.; Laird, N. \& Ware, J. H. (2012). Applied longitudinal analysis. 2 ed. New Jersey: John Wiley \& Sons.

Hebmuller, M. G.; Fiori, H. H. \& Lago, E. G. (2015). Gestações subsequentes em mulheres que tiveram sífilis na gestação. Ciência \& Saúde Coletiva, 20 (9): 2867-2878. http://dx.doi.org/10.1590/1413-81232015209.20332014.

Mccullagh, P. \& Nelder, J. A. (1989). Generalized linear models. New York: Chapman and Hall.

Moreira, K. F. A.; Oliveira, D. M.; Alencar, L. N.; Cavalcante, D. F. B.; Pinheiro, A. S. \& Orfão, N. H. (2017). Perfil dos casos notificados de sífilis congênita. Cogitare Enfermagem, 22 (2): e48949. http://dx.doi.org/10.5380/ce.v22i1.48949.

Newman, L.; Kamb, M.; Hawkes, S.; Gomez, G.; Say, L.; Seuc, A. et al. (2013). Global estimates of syphilis in pregnancy and associated adverse outcomes: analysis of multinational antenatal surveillance data. PLOS Medicine, 10(2):e1001396.

Nonato, S. M.; Melo, A. P. S. \& Guimarães, M. D. C. (2015). Sífilis na gestação e fatores associados à sífilis congênita em Belo Horizonte-MG, $2010-2013$. Epidemiologia \& Serviços de Saúde, 24 (4): 681-694. http://dx.doi.org/10.5123/S1679-49742015000400010.

Organização Pan-Americana da Saúde - OPAS. (2005). Plan estratégico regional de la Organización Panamericana de la Salud para el control de la infección por el VIH/SIDA y las infecciones de transmisión sexual (2006-2015) (resolução CD46.R15). 46 Conselho Diretor, 57 ${ }^{\circ}$ sessão do Comitê Regional da OMS para as Américas; 26 a 30 de setembro de 2005 . Washington (DC) EUA. Washington (DC). http://www.paho.org/hq/index.php?option=com_docman\&task=doc_download\&gid=18529\&Itemid=270\&lang=es.

Organização Mundial da Saúde (OMS). (2015). Orientaciones mundiales sobre los criterios y procesos para la validación de la eliminación de la transmisión maternoinfantil del VIH la OMS. https://apps.who.int/iris/bitstream/handle/10665/177835/9789243505886_spa.pdf;jsessionid=FD394DF12056F4D98FF3F260EC1E9595?sequence=1. 
Research, Society and Development, v. 10, n. 2, e30110212518, 2021

(CC BY 4.0) | ISSN 2525-3409 | DOI: http://dx.doi.org/10.33448/rsd-v10i2.12518

Paraná. (2021). Secretaria de Estado da Saúde do Paraná. TabNet. SINASC. Tabulação à partir de 1999. Nascidos vivos - Paraná - A partir de 1999. http://www.tabnet.sesa.pr.gov.br/tabnetsesa/dh?sistema/sinasc99diante/nascido.

Paraná. Secretaria de Estado da Saúde do Paraná. (2018). Linha guia rede mãe paranaense. 7 ed. Curitiba: Secretaria de Estado da Saúde do Paraná. p. 14, 25. http://www.saude.pr.gov.br/arquivos/File/LinhaGuiaMaeParanaense_2018.pdf.

Paraná. Secretaria de Estado da Saúde do Paraná. (2017). Manual para a gestão de caso na Rede Mãe Paranaense. Curitiba: Secretaria de Estado da Saúde do Paraná. p. 13. http://www.saude.pr.gov.br/arquivos/File/PRINTManual_Gestao_maeparanaense_15x21cm_25012018.pdf.

Pereira, A.S. et al. (2018). Metodologia da pesquisa científica. [e-book]. Santa Maria. Ed. UAB/NTE/UFSM. Recuperado de: em: https://repositorio.ufsm.br/bitstream/handle/1/15824/Lic_Computacao_Metodologia-Pesquisa-Cientifica.pdf?sequence=1

Pinheiro, J. M. \& Bates, D. M. (2000). Mixed-effects models in S and S-PLUS. New York: Springer-Verlag.

Rocha, R. P. \& Magajewski, F. R. L. (2018). Tendência histórico - epidemiológica da sífilis congênita no Estado de Santa Catarina no período $2007-2016$. Arquivos Catarinenses de Medicina, 47 (4): 39-52. http://www.acm.org.br/acm/seer/index.php/arquivos/article/view/350/297.

Secretaria de Estado da Saúde-SP. (2016). Centro de Controle de Doenças; Programa Estadual de DST/Aids. Centro de Referência e Treinamento DST/Aids. Organizadores: Luppi CG, Domingues CSB, Gomes SC. Guia de bolso para o manejo da sífilis em gestantes e sífilis congênita. 2 ed. São Paulo: Secretaria de Estado da Saúde. 112 p. http://www.saude.campinas.sp.gov.br/doencas/sifilis/guiadebolsodasifilis_2edicao2016.pdf.

Serruya, S. J.; Duran, P. Martinez, G.; Romero, M.; Caffe, S.; Alonso, M. \& Silveira, M. F. (2015). Maternal and congenital syphilis in selected Latin America and Caribbean countries: a multi-country analysis using data from the Perinatal Information System. Sexual Health, 12: 164-169. http://dx.doi.org/10.1071/SH14191.

Silveira, K. B. (2018). Epidemiologia e distribuição espacial da sífilis congênita em Sergipe. Dissertação (Mestrado em Biologia Parasitária). São Cristóvão: Universidade Federal de Sergipe, 122p. http://ri.ufs.br/jspui/handle/riufs/10150.

Slutsker, J. S.; Hennessy, R. R. \& Schillinger, J. A. (2018). Factors Contributing to Congenital Syphilis Cases - New York City, 2010-2016. Morbidity and Mortality Weekly Report, 67 (39): 1088-1093. https://www.cdc.gov/mmwr/volumes/67/wr/pdfs/mm6739a3-H.pdf.

Stamm, C.; Staduto, J. A. R.; Lima, J. F. \& Wadi, Y. M. (2013). A população urbana e a difusão das cidades de porte médio no Brasil. Interações, 14 (2): 251 265. http://www.scielo.br/pdf/inter/v14n2/a11v14n2.pdf.

Teixeira, L. O.; Belarmino, V.; Gonçalves, C. V. \& Mendoza-Sassi, R. A. (2018). Tendência temporal e distribuição espacial da sífilis congênita no estado do Rio Grande do Sul entre 2001 e 2012. Ciência \& Saúde Coletiva, 23 (8): 2587-2597. https://doi.org/10.1590/1413-81232018238.25422016. 